\title{
Detection of second-order topological superconductors by Josephson junctions
}

\author{
Song-Bo Zhang ${ }^{1}$ and Björn Trauzettel ${ }^{1,2}$ \\ ${ }^{1}$ Institut für Theoretische Physik und Astrophysik, Universität Würzburg, D-97074 Würzburg, Germany \\ ${ }^{2}$ Würzburg-Dresden Cluster of Excellence ct.qmat, Germany
}

(Received 23 May 2019; revised manuscript received 24 September 2019; published 16 January 2020)

\begin{abstract}
We study Josephson junctions based on second-order topological superconductors (SOTSs) which can be realized in quantum spin Hall insulators with a large inverted gap in proximity to unconventional superconductors. We find that tuning the chemical potential in the superconductor strongly modifies the induced pairing of the helical edge states, resulting in topological phase transitions. In a corresponding Josephson junction, a $0-\pi$ transition is realized by tuning the chemical potentials in the superconducting leads. This striking feature is stable in junctions with respect to different sizes, doping the normal region, and the presence of disorder. Our transport results can serve as novel experimental signatures of SOTSs. Moreover, the $0-\pi$ transition constitutes a fully electric way to create or annihilate Majorana bound states in the junction without any magnetic manipulation.
\end{abstract}

DOI: 10.1103/PhysRevResearch.2.012018

Introduction. The second-order topological superconductor (SOTS) is a novel topological phase of matter and features Majorana zero-dimensional (0D) corner or 1D hinge states which are two spatial dimensions lower than the gapped bulk [1-13]. They may form stable qubits for topological quantum computation [14-22]. Recently, the SOTS has been discovered in a variety of realistic materials and triggered tremendous interest [3-11,23-28]. One way to mimic SOTSs in $2 \mathrm{D}$ is given by quantum spin Hall insulators (QSHIs) in proximity to unconventional superconductors with $d_{x^{2}-y^{2}-}$ wave or $s_{ \pm}$-wave pairing order [3-5]. The proximity effect of unconventional superconductivity in $2 \mathrm{D}$ systems has been intensively explored in theory [29-38] and experiment [39-44]. To date, however, the only way proposed to detect 2D SOTSs is a tunneling experiment without a concrete calculation of the observable signature. An alternative approach to probe SOTSs and manipulate the Majorana corner modes is thus needed. In QSHIs, a finite doping is typically present, and the chemical potential can be far away from the Dirac points. Therefore, it is certainly interesting and experimentally relevant to explore the influence of the chemical potential in SOTSs.

In this Rapid Communication, we investigate superconductor-normal metal-superconductor (SNS) junctions formed by a 2D SOTS. The SOTS can be realized in a QSHI with a large inverted gap in proximity to an unconventional superconductor. We introduce a minimal model which is able to capture the essential physics of the SOTS. We find that due to the nontrivial momentum dependence of the pairing potential and mass, the chemical potential in the SOTS significantly alters the pairing gap opened within the edge states. It can even switch the sign of the pairing gap, leading to a

Published by the American Physical Society under the terms of the Creative Commons Attribution 4.0 International license. Further distribution of this work must maintain attribution to the author(s) and the published article's title, journal citation, and DOI. topological phase transition. While the supercurrent across the SNS junction is insensitive to the chemical potential in the $\mathrm{N}$ region, it depends strongly on the filling in the superconductors. Strikingly, tuning the chemical potentials in the superconductors gives rise to a $0-\pi$ transition, which is absent in junctions based on conventional $s$-wave pairing. These features are robust against disorder in junctions with novel sizes. They offer novel signatures to detect the SOTS with Majorana corner states. Furthermore, while Majorana bound states (MBSs) emerge in the 0 -junction when the phase difference across the junction is $\phi= \pm \pi$, they appear at vanishing $\phi$ in the $\pi$-junction. Thus, Josephson junctions with such a doping-induced $0-\pi$ transition provide an innovative platform to create or annihilate MBSs by electric gating in the absence of $\phi$. These predictions are applicable to a number of candidate systems including high-temperature QSHIs [45-56] in proximity to high-temperature cuprate or iron-based superconductors.

Minimal model for SOTSs. We consider the minimal model for SOTSs realized in QSHIs in proximity to superconductors,

$$
\begin{aligned}
H(\mathbf{k}) & =H_{0}(\mathbf{k})+\Delta(\mathbf{k}) \tau_{y} s_{y}, \\
H_{0}(\mathbf{k}) & =m(\mathbf{k}) \tau_{z} \sigma_{z}+v_{x} k_{x} s_{z} \sigma_{x}+v_{y} k_{y} \tau_{z} \sigma_{y}-\mu \tau_{z},
\end{aligned}
$$

written in the Nambu basis $\left(c_{a, \uparrow, \mathbf{k}}, c_{b, \uparrow, \mathbf{k}}, c_{a, \downarrow, \mathbf{k}}, c_{b, \downarrow, \mathbf{k}}, c_{a, \uparrow,-\mathbf{k}}^{\dagger}\right.$, $\left.c_{b, \uparrow,-\mathbf{k}}^{\dagger}, c_{a, \downarrow,-\mathbf{k}}^{\dagger}, c_{b, \downarrow,-\mathbf{k}}^{\dagger}\right)$, where $c_{\sigma, s, \mathbf{k}}^{\dagger} \quad\left(c_{\sigma, s, \mathbf{k}}\right) \quad$ creates (annihilates) an electron with spin $s \in\{\uparrow, \downarrow\}$, orbital $\sigma \in\{a, b\}$, and the momentum $\mathbf{k}=\left(k_{x}, k_{y}\right)$ measured from the band inversion point of the QSHI. $\tau, \sigma$, and $\boldsymbol{s}$ are Pauli matrices acting on Nambu, orbital, and spin spaces, respectively. $m(\mathbf{k})=m_{0}-m_{x} k_{x}^{2}-m_{y} k_{y}^{2}$ is the mass term of the QSHI and $\mu$ is the chemical potential. The band inversion implies the conditions $m_{0} m_{x}>0$ and $m_{0} m_{y}>0$ [57]. The pairing potential is written in general as $\Delta(\mathbf{k})=\Delta_{0}+\Delta_{2}\left(k_{x}^{2}-k_{y}^{2}\right)$. When $\Delta_{0} \neq 0$ and $\Delta_{2}=0$, it refers to conventional $s$-wave pairing. When $\Delta_{0}=0$ and $\Delta_{2} \neq 0$, the pairing is formally $d_{x^{2}-y^{2}}$ wave. It can 
be induced in a QSHI with band inversion at the $\Gamma$ point via the proximity to a cuprate superconductor [4]. When $0 \leqslant\left|\Delta_{0}\right|<m_{0}\left|\Delta_{2}\right| / 2 m_{x(y)}$, the system possesses a mixture of $s$-wave and $d_{x^{2}-y^{2}}$-wave pairing. It can also describe effectively a QSHI with band inversion at the $X$ point [54-56] and $s_{ \pm}$-wave pairing induced from an iron-based superconductor [58-62].

In the absence of $\Delta(\mathbf{k})$, the system hosts gapless helical edge states across the bulk gap, which are protected by timereversal symmetry. The pairing term with $\Delta_{2} \neq 0$ induces a pairing gap of the edge states. The gap may switch sign at the corners, resulting in Majorana corner modes [3-5]. We note that although the model (1) is a low-energy effective model, it captures the essential physics of the SOTS. Based on this model, we can understand the second-order topology more intuitively from the picture of edge states and show that it can be strongly altered by changing $\mu$.

Pairing gaps of edge states and topological phase transitions. To analyze the Majorana corner states and the influence of $\mu$ on the SOTS, we analytically derive the effective model for edge states. For illustration, we consider the edge along the $x$ direction of the SOTS in the half plane $y \leqslant 0$ and assume hard-wall boundary conditions [63]. As in realistic systems, we assume weak pairing. We first calculate the edge states of $H_{0}$, following the approach of Ref. [64]. In this model, $k_{x}$ is a good quantum number. The helical electron and hole edge bands are found explicitly as

$$
E_{e(h), \uparrow \downarrow}\left(k_{x}\right)= \pm \operatorname{sgn}\left(m_{y} v_{y}\right) v_{x} k_{x}-(+) \mu .
$$

The wave functions in the orbital basis $\{a, b\}$ read

$$
\Psi_{e, \uparrow, k_{x}}(\mathbf{r})=\mathcal{N} e^{i k_{x} x}\left(e^{\lambda_{1} y}-e^{\lambda_{2} y}\right)\left[\operatorname{sgn}\left(m_{y} v_{y}\right), 1\right]^{T} .
$$

They fulfill $\Psi_{e, \downarrow, k_{x}}(\mathbf{r})=\Psi_{e, \uparrow,-k_{x}}^{*}(\mathbf{r})$ and $\Psi_{h, \downarrow / \uparrow, k_{x}}(\mathbf{r})=$ $\Psi_{e, \downarrow / \uparrow,-k_{x}}^{*}(\mathbf{r})$, due to time-reversal and particle-hole symmetries; $\quad \lambda_{1(2)}=\left|v_{y} / 2 m_{y}\right|-(+)\left(v_{y}^{2} / 4 m_{y}^{2}-m_{0} / m_{y}+m_{x} k_{x}^{2} /\right.$ $\left.m_{y}\right)^{1 / 2}$ and $\mathcal{N}$ is the normalization factor. The decaying length of edge states is given by $\xi_{\text {edge }}=\max \left[1 / \operatorname{Re}\left(\lambda_{1}\right), 1 / \operatorname{Re}\left(\lambda_{2}\right)\right]$. At zero energy, the electron and hole bands touch at $k_{x}= \pm k_{c}$ with $k_{c}=\operatorname{sgn}\left(m_{y} v_{y}\right) \mu / v_{x}$. For $\mu=0, k_{c}=0$. However, for $\mu \neq 0$, the touching points shift to finite $\pm k_{c}$. Projecting the pairing term onto the edge states, the resulting Bogoliubov-de Gennes $(\mathrm{BdG})$ Hamiltonian for edge states is obtained as

$$
h_{\mathrm{BdG}}^{x}=\operatorname{sgn}\left(m_{y} v_{y}\right) v_{x} k_{x} \tau_{z} s_{z}-\mu \tau_{z}+\Delta_{\mathrm{eff}}^{x} \tau_{x} s_{z}
$$

in the basis $\left(\Psi_{e, \uparrow}, \Psi_{e, \downarrow}, \Psi_{h, \downarrow}, \Psi_{h, \uparrow}\right)$, and the pairing gap is given by

$$
\Delta_{\text {eff }}^{x}=-\Delta_{0}+\Delta_{2}\left[m_{0} / m_{y}-\left(1+m_{x} / m_{y}\right) \mu^{2} / v_{x}^{2}\right] .
$$

Without loss of generality, a real $\Delta(\mathbf{k})$ has been assumed [65]. We provide the derivation in detail in the Supplemental Material [66]. Similarly, for an edge along the $y$ direction, we find the BdG Hamiltonian of the same form but with a different pairing gap,

$$
\Delta_{\text {eff }}^{y}=-\Delta_{0}-\Delta_{2}\left[m_{0} / m_{x}-\left(1+m_{y} / m_{x}\right) \mu^{2} / v_{y}^{2}\right] .
$$

The combination of $\Delta_{\text {eff }}^{x}$ and $\Delta_{\text {eff }}^{y}$ (with opposite signs) in Eq. (4) mimics the Jackiw-Rebbi model [67] at the corners of the $x$ and $y$ axes. Thus, Majorana corner states at zero energy appear if $\Delta_{\text {eff }}^{x} \Delta_{\text {eff }}^{y}<0$.
For $s$-wave pairing, $\Delta_{\text {eff }}^{x}$ and $\Delta_{\text {eff }}^{y}$ are identical and constant. Thus, no corner state emerges. In contrast, for unconventional pairings with $\left|\Delta_{0}\right|<m_{0}\left|\Delta_{2}\right| / 2 m_{x(y)}$, we obtain corner states at small $\mu$. When $\mu=0$, the SOTS has two reflection symmetries. When $m_{x}=m_{y}, v_{x}=v_{y}$, and $\Delta_{0}=0$, it possesses a fourfold rotation symmetry. In these particular cases, the system can be characterized by a topological invariant calculated from the bulk Hamiltonian [66,68-70]. However, the corner states in our model are not restricted to any crystalline symmetries. Interestingly, $\Delta_{\text {eff }}^{x(y)}$ depends strongly on $\mu$. The $\mu$ dependence stems from the quadratic terms in the model (1), which are crucial for the topological properties of the SOTS. Moreover, $\Delta_{\mathrm{eff}}^{x(y)}$ vanishes at $\mu= \pm \mu_{x(y)}^{c}$, where

$$
\mu_{x(y)}^{c}=\left|v_{x(y)}\right| \sqrt{\left[m_{0} / m_{y(x)} \mp \Delta_{0} / \Delta_{2}\right] /\left(1+m_{x(y)} / m_{y(x)}\right)} .
$$

This behavior indicates that we can switch the sign of $\Delta_{\text {eff }}^{x(y)}$ by varying $\mu$. Without loss of generality, we suppose $\mu_{x}^{c} \leqslant \mu_{y}^{c}$. The system is in a SOTS phase in the parameter regions $0 \leqslant|\mu|<\mu_{x}^{c}$ and $\mu_{y}^{c}<|\mu|<m_{\mathrm{g}}$ with $m_{\mathrm{g}}$ being the bulk gap [71], whereas if $\mu_{x}^{c}<|\mu|<\mu_{y}^{c}$, it becomes a trivial superconductor with no corner state. For the particular case with $v_{x(y)}=v, m_{x(y)}=m$, and $\Delta_{0}=0, \Delta_{\text {eff }}^{x}$ and $\Delta_{\text {eff }}^{y}$ are always opposite. They both close at $\mu= \pm v \sqrt{m_{0} / 2 m}$. Thus, there is no parameter space for the trivial phase. Nevertheless, the sign of $\Delta_{\text {eff }}^{x(y)}$ can still be changed by a finite $\mu$ inside the bulk gap [72] if

$$
2 m_{0} m>v^{2} .
$$

This condition indeed corresponds to a QSHI phase with a large inverted gap or equivalently an indirect bulk gap. It is likely realized in the inverted InAs/GaSb bilayer [73-75], $\mathrm{WTe}_{2}$ monolayer [45-49], functionalized MXene [50,51], bismuthene on $\mathrm{SiC}$ [52,53], and $\mathrm{PbS}$ monolayer [54-56].

To test our analytical results, we discretize the model (1), put it on a square lattice, choose a proper set of parameters [satisfying the inequality (8)], and calculate the energy spectrum in a ribbon geometry [76]. For concreteness, we consider $\Delta_{0}=0$ and set the lattice constant $a$ to unity. As shown in Fig. 1(a), the edge states for $\mu=0$ open a gap at $k_{x}=0$. As $\mu$ is increased, the gap splits to two points away from $k_{x}=0$. The magnitude of the gap first decreases, vanishes at a critical $\mu$, and then reopens, which explicitly demonstrates a topological phase transition. This behavior is in perfect agreement with Eq. (5) [cf. Fig. 1(b)].

$0-\pi$ transition and its robustness. We now consider an SNS junction in which two SOTSs (also called S leads below) are connected by a QSHI with length $L$ in the $x$ direction, as sketched in Fig. 2(a). The width of the junction ribbon is $W$. For simplicity, we assume the chemical and pairing potentials in steplike forms. $\mu_{L(R)}$ and $\mu_{N}$ denote the chemical potentials in the left (right) $\mathrm{S}$ lead and $\mathrm{N}$ (QSHI) region, respectively. $\phi$ is the phase difference across the junction. We calculate the supercurrent $J_{s}$ by the lattice Green's function technique [77-79] and provide the details in the Supplemental Material [66].

At low temperatures, the transport in the junction is conducted dominantly by helical edge channels. Perfect Andreev reflection occurs at the NS interfaces. Thus, the current-phase relation (CPR) takes a sawtooth shape with a sudden jump 

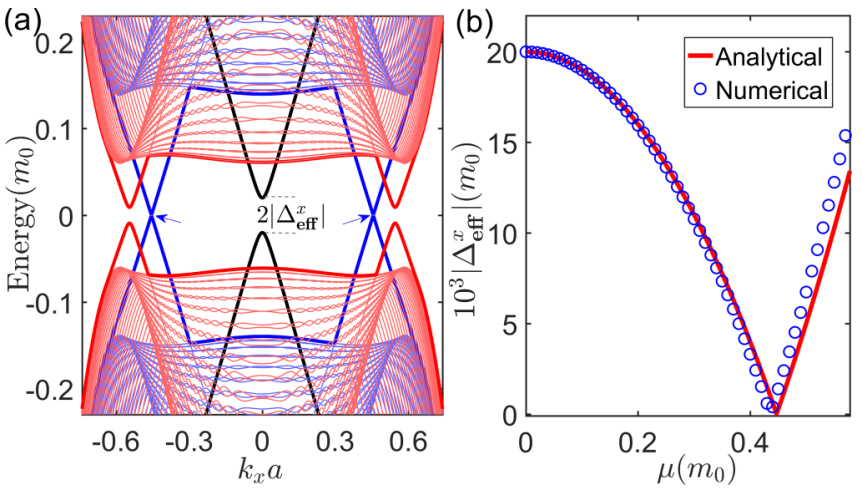

FIG. 1. (a) Energy spectra of the model (1) in a ribbon along the $x$ direction for chemical potential $\mu=0$ (black), $0.44 m_{0}$ (blue), and $0.52 m_{0}$ (red), respectively. The thick curves close to zero energy are edge bands. The pairing gap $\Delta_{\text {eff }}^{x}$ vanishes at around $\mu=0.44 m_{0}$, as pointed by the blue arrows. (b) $\left|\Delta_{\text {eff }}^{x}\right|$ as a function of $\mu$. The blue circles are numerical results from tight-binding calculation while the red curve is the plot of Eq. (5). Other parameters are $m_{x(y)}=$ 2.5, $v_{x(y)}=1, \Delta_{0}=0, \Delta_{2}=0.05$, and 200 lattice layers in the $y$ direction. The units for energy and wave number are $m_{0}$ and $a^{-1}$, respectively.

[see Fig. 2(b)]. The sawtoothlike CPR is insensitive to $\mu_{N}$ and stays stable in junctions of different sizes ( $L$ and $W$ ), provided that the two edges at $y= \pm W / 2$ are well separated, $W \gg \xi_{\text {edge }}$. The sudden jump can be related to the fermion parity anomaly at each edge [80,81]. It indicates the formation of degenerate MBSs in the junction discussed below. $J_{s}$ decreases monotonically with increasing $L$ [see Fig. 2(b)]. The critical current $J_{c}$ (maximal value of $J_{s}$ ) decays as $\sim 1 / L$
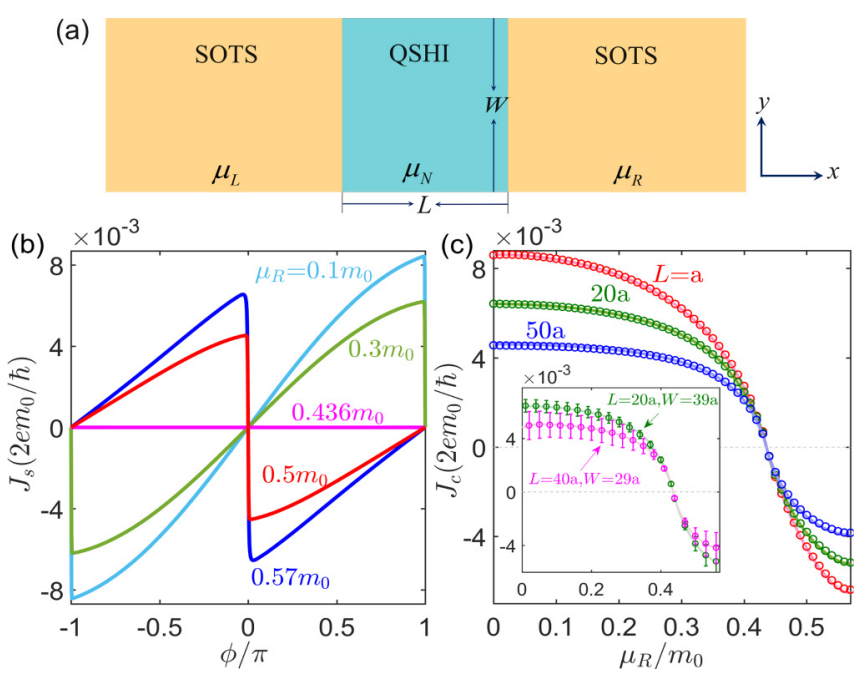

FIG. 2. (a) Schematic for the SNS setup. (b) Current-phase relations for $L=a$ and $\mu_{R}=0.1 m_{0}, 0.3 m_{0}, 0.436 m_{0}, 0.5 m_{0}$, and $0.57 m_{0}$, respectively. (b) Critical current $J_{c}$ as a function of $\mu_{R}$ for $L=a$ (red), $20 a$ (green), and 50a (blue), respectively. The inset displays the results in the presence of disorder of strength $V_{\text {dis }}=m_{0}$ for $W=39 a$ and $L=20 a$ (green), $W=29 a$ and $L=40 a$ (purple), respectively. The error bars are 500 times enlarged for visibility. For all solid curves, $\mu_{L}=\mu_{N}=0.1 m_{0}, W=39 a, k_{B} T=10^{-3} \Delta_{L}$, and other parameters are the same as those in Fig. 1. The circled dots in (c) are the same as the solid curves but for $\mu_{N}=-0.3 m_{0}$. in long junctions, similar to junctions based on conventional $s$-wave pairing. In short junctions, $J_{c}$ is of the same order of magnitude but always smaller than $e \sqrt{\left|\Delta_{L} \Delta_{R}\right|} / \hbar$, in contrast to the case of $s$-wave pairing. In this estimate, $\Delta_{L(R)}$ is the induced pairing gap of edge states in the left (right) $\mathrm{S}$ lead and determined by Eq. (5). We attribute this difference to the inhomogeneity of the superconducting pairing at the boundaries of our setup.

The CPRs for a fixed $\mu_{L}$ and various values of $\mu_{R}$ are displayed in Fig. 2(a). Since $J_{s}$ is even in $\mu_{L(R)}$, we present only the results for $\mu_{L(R)}>0$. While $J_{S}$ is insensitive to $\mu_{N}$, it decreases significantly when we increase $\mu_{R}$. This behavior can be understood as a result of the reduction of $\left|\Delta_{R}\right|$ by $\mu_{R}$ [see Eq. (5)]. Strikingly, increasing $\mu_{R}$ further, we observe a clear $0-\pi$ transition for the parameters satisfying the inequality (8). While $J_{s}(\phi)$ in the region $0<\phi<\pi$ is positive for $\mu_{R}<\mu^{c}$, it becomes negative for $\mu_{R}>\mu^{c}$. We coin the former case a 0 -junction and the latter one a $\pi$ junction. Meanwhile, the sudden jump of the CPR is switched to $\phi=0$ in the $\pi$-junction, which is in strong contrast to the 0 -junction where the jump is at $\phi= \pm \pi$. In Fig. 2(c), we plot $J_{c}$ as a function of $\mu_{R}$. The critical value $\mu^{c}$ for the transition is approximately given by $v \sqrt{m_{0} / 2 m}$, in accord with our analytical result. Close to $\mu^{c}, J_{c}$ drops quickly and switches sign. These features are generic and apply to junctions of different lengths and widths. They are also robust with respect to nonmagnetic disorder in the $\mathrm{N}$ region. To illustrate this, we model the disorder as random on-site potentials in the range $\left[-V_{\mathrm{dis}} / 2, V_{\mathrm{dis}} / 2\right][66,82]$ and calculate 200 random disorder configurations in the inset of Fig. 2(c). There is no qualitative difference in the features compared to those in clean junctions. This can be expected since the helical edge channels which mediate the transport are less sensitive to backscattering. Similar effects can be observed by tuning $\mu_{L}$ and fixing $\mu_{R}$. Finally, it is important to note that the variations of $J_{S}$ and the $0-\pi$ transition by tuning $\mu_{L(R)}$ are directly related to the strong $\mu_{L(R)}$ dependence in $\Delta_{L(R)}$ in the SOTS, and absent in conventional junctions based on $s$-wave pairing.

Majorana bound states. Next, we discuss the Andreev bound states (ABSs) formed in the junction, which can be obtained from the lattice Green's function. In short junctions, there are two bands of ABSs with opposite energies [see Figs. 3(a) and 3(c)]. When the sudden jump of the CPR occurs, the positive and negative bands touch at zero energy. This degeneracy is robust and protected by time-reversal and particle-hole symmetries. It resembles Kramers pairs of MBSs. This can be best understood from the effective Hamiltonian (4) for edge states. In the short-junction limit, two ABS bands at a given edge can be described by

$$
E_{ \pm}(\phi)= \pm \frac{\Delta_{L} \Delta_{R} \sin \phi}{\sqrt{\Delta_{L}^{2}+\Delta_{R}^{2}-2 \Delta_{L} \Delta_{R} \cos \phi}} .
$$

Notably, the ABSs are confined in the pairing gaps for $\phi$ satisfying $\left(\cos \phi-\Delta_{L} / \Delta_{R}\right)\left(\cos \phi-\Delta_{R} / \Delta_{L}\right)>0$, as verified in Figs. 3(a) and 3(c). Noticing $\Delta_{L} \Delta_{R}>0$ in the 0-junction for $\mu_{R}<\mu^{c}$, whereas $\Delta_{L} \Delta_{R}<0$ in the $\pi$-junction for $\mu_{R}>$ $\mu^{c}$, we can see that $E_{ \pm}(\phi)$ touch at $\phi= \pm \pi$ and 0 , respectively. Using the valid formula at zero temperature, $J_{s}(\phi)=$ $\partial\left|E_{+}(\phi)\right| / \partial \phi[83]$, we also reproduce the sudden jump in the 


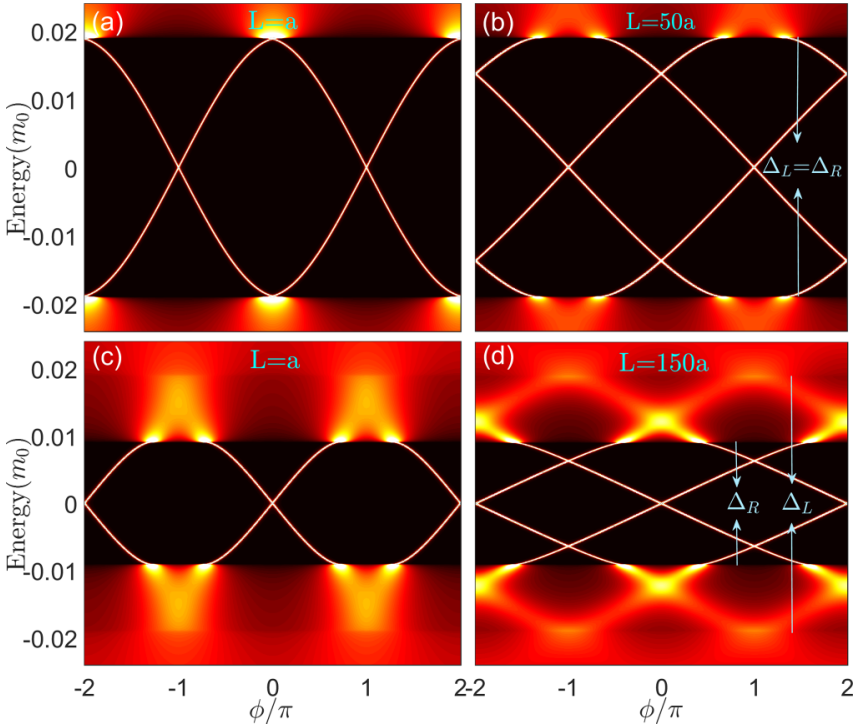

FIG. 3. Andreev bound states for (a), (b) $\mu_{R}=0.1 m_{0}$ and (c), (d) $\mu_{R}=0.52 m_{0}$, respectively. (a) and (c) are for short junctions with $L=a$, while (b) and (d) for long junctions with $L=50 a$ and $150 a$, respectively. For all plots, $W=39 a$ and other parameters are the same as those in Fig. 1.

CPR. The wave functions of the zero modes can be written as

$$
\begin{aligned}
& \gamma_{+}(x)=\Psi_{+} \eta(x)+\Psi_{-} \eta^{*}(x), \\
& \gamma_{-}(x)=i \Psi_{+} \eta(x)-i \Psi_{-} \eta^{*}(x),
\end{aligned}
$$

where $\Psi_{ \pm}=\operatorname{sgn}\left(\Delta_{L}\right) \Psi_{h / e, \downarrow} \mp i \Psi_{e / h, \uparrow}$ and the spatial dependence is $\eta(x)=\exp \left\{\left[\theta(x)\left(i \mu_{R}-\left|\Delta_{R}\right|\right)+\theta(-x)\left(i \mu_{L}+\right.\right.\right.$ $\left.\left.\left.\left|\Delta_{L}\right|\right)\right] x / v\right\}$ [66]. Since $\Psi_{h, \downarrow / \uparrow}=\Psi_{e, \downarrow / \uparrow}^{*}$, the zero modes have self-adjoint wave functions, $\gamma_{ \pm}(x)=\gamma_{ \pm}^{*}(x)$. They are Majorana fermions. Under the time-reversal operation $\mathcal{T}$, $\mathcal{T} \Psi_{e, \downarrow / \uparrow}= \pm \Psi_{e \uparrow / \downarrow}^{*}$ and $\mathcal{T} \Psi_{h, \downarrow / \uparrow}= \pm \Psi_{h, \uparrow / \downarrow}^{*}$. Therefore, $\gamma_{ \pm}$ are related by time-reversal symmetry, $\mathcal{T} \gamma_{+}=\gamma_{-}$. A similar analysis can be applied to the other edge where another Kramers pair of MBSs is located. In long junctions, all the features persist but with more pairs of discrete ABS bands emerging from the continuum spectrum [see Figs. 3(b) and 3(d)].

At $\phi=0$, the MBSs emerge for $\mu>\mu^{c}$, whereas they disappear for $\mu<\mu^{c}$. In this sense, we are able to switch between the presence and absence of MBSs by gating the $S$ leads in the absence of $\phi$. Our setup indeed realizes fully electrically controllable MBSs without fine tuning of the magnetic field or threaded flux. This is an important advantage compared to previous proposals based on conventional $s$ wave superconductivity [24,80,84-87]. Moreover, since the localization lengths of the MBSs in the $\mathrm{S}$ leads are determined by $\xi_{L(R)}=\left|v / \Delta_{L(R)}\right|$, we are also able to control the spatial profiles of the MBSs by $\mu_{L(R)}$.

Experimental relevance and summary. Now we briefly discuss the experimental relevance of our proposal. QSHIs with large inverted gaps [45-56,73-75] in proximity to cuprate or iron-based superconductors [39-41,58-62] could provide promising platforms to verify our predictions. For concreteness, we take the inverted InAs/GaSb bilayer and $\mathrm{WTe}_{2}$ monolayer to estimate $\mu_{x(y)}^{c}$. For simplicity, we consider $\Delta_{0}=$ 0 such that $\mu_{x}^{c}(y)$ is independent of the magnitude of the pairing potential. For the inverted InAs/GaSb bilayer, $m_{0}=$ $0.0055 \mathrm{eV}, m_{x(y)}=81.9 \mathrm{eV} \AA^{2}, v_{x(y)}=0.72 \mathrm{eV} \AA$ [88]. To realize the $0-\pi$ transition, one can fabricate the Josephson junction in any direction and find that $\mu_{x(y)}^{c}=0.0042 \mathrm{eV}$ which is smaller than the bulk gap $m_{\text {gap }}=0.005 \mathrm{eV}$. For the $\mathrm{WTe}_{2}$ monolayer with $m_{0}=0.33 \mathrm{eV}, m_{x}=4.6 \mathrm{eV} \AA^{2}$, $m_{y}=16.9 \mathrm{eV} \AA^{2}, v_{x}=2.55 \mathrm{eV} \AA$, and $v_{y}=0.3 \mathrm{eV} \AA$ [45], we have $\mu_{x}^{c}=0.252 \mathrm{eV}, \mu_{y}^{c}=0.057 \mathrm{eV}$, and $m_{\text {gap }}=0.08 \mathrm{eV}$. Thus, it is better to design the junction in the $y$ direction in our model [89]. According to Eq. (7), the inclusion of a small $\Delta_{0}$ would suppress $\mu_{x}^{c}$ or $\mu_{y}^{c}$ and hence make it more feasible to observe the $0-\pi$ transition. A particle-hole symmetry breaking term, which is neglected here, breaks the symmetry with respect to $\mu$ but does not qualitatively change our main results.

We note in passing that there have been experimental efforts trying to incorporate unconventional superconductivity in topological systems [39-41,60-62]. Moreover, large proximity-induced pairing gaps in $2 \mathrm{D}$ systems from unconventional superconductors have been probed [39-42].

In summary, we have found that the chemical potentials in superconductors can be used to modulate the supercurrent and realize a $0-\pi$ transition in Josephson junctions based on SOTSs. These features are attributed to the dependence of the pairing gap of edge states on the chemical potential. They could serve as novel experimental signatures of the SOTS. We have predicted the $0-\pi$ transition as a fully electric way to create or annihilate MBSs at elevated temperatures.

Acknowledgments. We thank Fernando Dominguez, Feng Liu, Frank Schindler, Gaomin Tang, Xianxin Wu, and Wenbin Rui for valuable discussion. This work was supported by the DFG (SPP1666 and SFB1170 "ToCoTronics"), the Würzburg-Dresden Cluster of Excellence ct.qmat, EXC2147, Project ID 39085490, and the Elitenetzwerk Bayern Graduate School on "Topological Insulators."
[1] J. Langbehn, Y. Peng, L. Trifunovic, F. von Oppen, and P. W. Brouwer, Reflection-Symmetric Second-Order Topological Insulators and Superconductors, Phys. Rev. Lett. 119, 246401 (2017).

[2] E. Khalaf, Higher-order topological insulators and superconductors protected by inversion symmetry, Phys. Rev. B 97, 205136 (2018).
[3] Q. Wang, C.-C. Liu, Y.-M. Lu, and F. Zhang, High-Temperature Majorana Corner States, Phys. Rev. Lett. 121, 186801 (2018).

[4] Z. Yan, F. Song, and Z. Wang, Majorana Corner Modes in a High-Temperature Platform, Phys. Rev. Lett. 121, 096803 (2018).

[5] T. Liu, J. J. He, and F. Nori, Majorana corner states in a two-dimensional magnetic topological insulator on a 
high-temperature superconductor, Phys. Rev. B 98, 245413 (2018).

[6] M. Geier, L. Trifunovic, M. Hoskam, and P. W. Brouwer, Second-order topological insulators and superconductors with an order-two crystalline symmetry, Phys. Rev. B 97, 205135 (2018).

[7] X. Zhu, Tunable Majorana corner states in a two-dimensional second-order topological superconductor induced by magnetic fields, Phys. Rev. B 97, 205134 (2018).

[8] Y. Wang, M. Lin, and T. L. Hughes, Weak-pairing higher order topological superconductors, Phys. Rev. B 98, 165144 (2018).

[9] C.-H. Hsu, P. Stano, J. Klinovaja, and D. Loss, Majorana Kramers Pairs in Higher-Order Topological Insulators, Phys. Rev. Lett. 121, 196801 (2018).

[10] R.-X. Zhang, W. S. Cole, and S. Das Sarma, Helical Hinge Majorana Modes in Iron-Based Superconductors, Phys. Rev. Lett. 122, 187001 (2019).

[11] S. Qin, L. Hu, C. Le, J. Zeng, F.-C. Zhang, C. Fang, and J. $\mathrm{Hu}$, Quasi 1D Topological Nodal Vortex Line Phase in Doped Superconducting 3D Dirac Semimetals, Phys. Rev. Lett. 123, 027003 (2019).

[12] N. Bultinck, B. A. Bernevig, and M. P. Zaletel, Threedimensional superconductors with hybrid higher-order topology, Phys. Rev. B 99, 125149 (2019).

[13] Y. Peng and Y. Xu, Proximity-induced Majorana hinge modes in antiferromagnetic topological insulators, Phys. Rev. B 99, 195431 (2019).

[14] A. Y. Kitaev, Unpaired Majorana fermions in quantum wires, Phys. Usp. 44, 131 (2001).

[15] A Y. Kitaev, Fault-tolerant quantum computation by anyons, Ann. Phys. 303, 2 (2003).

[16] C. Nayak, S. H. Simon, A. Stern, M. Freedman, and S. Das Sarma, Non-Abelian anyons and topological quantum computation, Rev. Mod. Phys. 80, 1083 (2008).

[17] J. Alicea, New directions in the pursuit of Majorana fermions in solid state systems, Rep. Prog. Phys. 75, 076501 (2012).

[18] M. Leijnse and K. Flensberg, Introduction to topological superconductivity and Majorana fermions, Semicond. Sci. Technol. 27, 124003 (2012).

[19] C. W. J. Beenakker, Search for Majorana Fermions in Superconductors, Annu. Rev. Condens. Matter Phys. 4, 113 (2013).

[20] S. R. Elliott and M. Franz, Colloquium: Majorana fermions in nuclear, particle, and solid-state physics, Rev. Mod. Phys. 87, 137 (2015).

[21] S. Das Sarma, M. Freedman, and C. Nayak, Majorana zero modes and topological quantum computation, npj Quantum Inf. 1, 15001 (2015).

[22] M. Sato and S. Fujimoto, Majorana fermions and topology in superconductors, J. Phys. Soc. Jpn. 85, 072001 (2016).

[23] E. J. König and P. Coleman, Helical Majorana Modes in Iron Based Dirac Superconductors, Phys. Rev. Lett. 122, 207001 (2019).

[24] Y. Volpez, D. Loss, and J. Klinovaja, Second-Order Topological Superconductivity in $\pi$-Junction Rashba Layers, Phys. Rev. Lett. 122, 126402 (2019).

[25] H. Shapourian, Y. Wang, and S. Ryu, Topological crystalline superconductivity and second-order topological superconductivity in nodal-loop materials, Phys. Rev. B 97, 094508 (2018).

[26] X.-H. Pan, K.-J. Yang, L. Chen, G. Xu, C.-X. Liu, and X. Liu, Lattice Symmetry Assisted Second Order Topological
Superconductors and Majorana Patterns, Phys. Rev. Lett. 123, 156801 (2019).

[27] M. Kheirkhah, Y. Nagai, C. Chen, and F. Marsiglio, Majorana corner flat bands in two-dimensional second-order topological superconductors, arXiv:1904.00990.

[28] S. A. A. Ghorashi, X. Hu, T. L. Hughes, and E. Rossi, Secondorder Dirac superconductors and magnetic field induced Majorana hinge modes, Phys. Rev. B 100, 020509 (2019).

[29] J. Linder and A. Sudbø, Tunneling conductance in $s$ - and $d$-wave superconductor-graphene junctions: Extended BlonderTinkham-Klapwijk formalism, Phys. Rev. B 77, 064507 (2008).

[30] J. Linder, Y. Tanaka, T. Yokoyama, A. Sudb $\varnothing$, and N. Nagaosa, Unconventional Superconductivity on a Topological Insulator, Phys. Rev. Lett. 104, 067001 (2010).

[31] A. M. Black-Schaffer and A. V. Balatsky, Proximity-induced unconventional superconductivity in topological insulators, Phys. Rev. B 87, 220506(R) (2013).

[32] F. Zhang, C. L. Kane, and E. J. Mele, Time-Reversal-Invariant Topological Superconductivity and Majorana Kramers Pairs, Phys. Rev. Lett. 111, 056402 (2013).

[33] Z.-X. Li, C. Chan, and H. Yao, Realizing majorana zero modes by proximity effect between topological insulators and $d$-wave high-temperature superconductors, Phys. Rev. B 91, 235143 (2015).

[34] P. Zareapour, J. Xu, S. Yang F. Zhao, A. Jain, Z. Xu, T. S. Liu, G. D. Gu, and K. S. Burch, Modeling tunneling for the unconventional superconducting proximity effect, Supercond. Sci. Technol. 29, 125006 (2016).

[35] W.-J. Li, S.-P. Chao, and T.-K. Lee, Theoretical study of large proximity-induced $s$-wave-like pairing from a $d$-wave superconductor, Phys. Rev. B 93, 035140 (2016).

[36] X. Wu, S. Qin, Y. Liang, H. Fan, and J. Hu, Topological characters in $\mathrm{Fe}\left(\mathrm{Te}_{1-x} \mathrm{Se}_{x}\right)$ thin films, Phys. Rev. B 93, 115129 (2016).

[37] Z. Wang, P. Zhang, G. Xu, L. K. Zeng, H. Miao, X. Xu, T. Qian, H. Weng, P. Richard, A. V. Fedorov, H. Ding, X. Dai, and Z. Fang, Topological nature of the $\mathrm{FeSe}_{0.5} \mathrm{Te}_{0.5}$ superconductor, Phys. Rev. B 92, 115119 (2015).

[38] T. Zhou, Y. Gao, and Z. D. Wang, Detecting competing orders through the edge states in the heterostructures with high- $T_{c}$ superconductors, Phys. Rev. B 99, 104517 (2019).

[39] P. Zareapour, A. Hayat, S. Y. F. Zhao, M. Kreshchuk, A. Jain, D. C. Kwok, N. Lee, S.-W. Cheong, Z. Xu, A. Yang et al., Proximity-induced high-temperature superconductivity in the topological insulators $\mathrm{Bi}_{2} \mathrm{Se}_{3}$ and $\mathrm{Bi}_{2} \mathrm{Te}_{3}$, Nat. Commun. 3, 1056 (2012).

[40] E. Wang, H. Ding, A. V. Fedorov, W. Yao, Z. Li, Y.-F. Lv, K. Zhao, L.-G. Zhang, Z. Xu, J. Schneeloch et al., Fully gapped topological surface states in $\mathrm{Bi}_{2} \mathrm{Se}_{3}$ films induced by a $d$-wave high-temperature superconductor, Nat. Phys. 9, 621 (2013).

[41] H. Zhao, B. Rachmilowitz, Z. Ren, R. Han, J. Schneeloch, R. Zhong, G. Gu, Z. Wang, and I. Zeljkovic, Superconducting proximity effect in a topological insulator using $\mathrm{Fe}(\mathrm{Te}, \mathrm{Se})$, Phys. Rev. B 97, 224504 (2018).

[42] D. Perconte, F. A. Cuellar, C. Moreau-Luchaire, M. PiquemalBanci, R. Galceran, P. R Kidambi, M.-B. Martin, S. Hofmann, R. Bernard, B. Dlubak et al., Tunable Klein-like tunneling of high-temperature superconducting pairs into graphene, Nat. Phys. 14, 25 (2018). 
[43] S.-Y. Xu, C. Liu, A. Richardella, I. Belopolski, N. Alidoust, M. Neupane, G. Bian, N. Samarth, and M. Z. Hasan, Fermilevel electronic structure of a topological-insulator/cupratesuperconductor based heterostructure in the superconducting proximity effect regime, Phys. Rev. B 90, 085128 (2014).

[44] T. Yilmaz, I. Pletikosić, A. P. Weber, J. T. Sadowski, G. D. Gu, A. N. Caruso, B. Sinkovic, and T. Valla, Absence of a Prox-

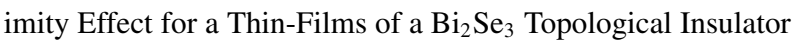
Grown on Top of $\mathrm{a}_{2} \mathrm{Sr}_{2} \mathrm{CaCu}_{2} \mathrm{O}_{8+\delta}$ Cuprate Superconductor, Phys. Rev. Lett. 113, 067003 (2014).

[45] X. Qian, J. Liu, L. Fu, and J. Li, Quantum spin Hall effect in two-dimensional transition metal dichalcogenides, Science 346, 1344 (2014).

[46] S. Tang, C. Zhang, D. Wong, Z. Pedramrazi, H.-Z. Tsai, C. Jia, B. Moritz, M. Claassen, H. Ryu, S. Kahn et al., Quantum spin Hall state in monolayer $1 T^{\prime}-\mathrm{WTe}_{2}$, Nat. Phys. 13, 683 (2017).

[47] Z. Fei, T. Palomaki, S. Wu, W. Zhao, X. Cai, B. Sun, P. Nguyen, J. Finney, X. Xu, and D. H. Cobden, Edge conduction in monolayer $\mathrm{WTe}_{2}$, Nat. Phys. 13, 677 (2017).

[48] S. Wu, V. Fatemi, Q. D. Gibson, K. Watanabe, T. Taniguchi, R. J. Cava, and P. Jarillo-Herrero, Observation of the quantum spin Hall effect up to 100 kelvin in a monolayer crystal, Science 359, 76 (2018).

[49] P Chen, W. W. Pai, Y.-H. Chan, W.-L. Sun, C.-Z. Xu, D.-S. Lin, M. Y. Chou, A.-V. Fedorov, and T.-C. Chiang, Large quantumspin-Hall gap in single-layer $1 T^{\prime}-\mathrm{WSe}_{2}$, Nat. Commun. 9, 2003 (2018).

[50] H. Weng, A. Ranjbar, Y. Liang, Z. Song, M. Khazaei, S. Yunoki, M. Arai, Y. Kawazoe, Z. Fang, and X. Dai, Large-gap two-dimensional topological insulator in oxygen functionalized MXene, Phys. Rev. B 92, 075436 (2015).

[51] C. Si, K.-H. Jin, J. Zhou, Z. Sun, and F. Liu, Large-gap quantum spin Hall state in MXenes: $d$-band topological order in a triangular lattice, Nano Lett. 16, 6584 (2016).

[52] F. Reis, G. Li, L. Dudy, M. Bauernfeind, S. Glass, W. Hanke, R. Thomale, J. Schäfer, and R. Claessen, Bismuthene on a SiC substrate: A candidate for a high-temperature quantum spin Hall material, Science 357, 287 (2017).

[53] C.-H. Hsu, Z.-Q. Huang, F.-C. Chuang, C.-C. Kuo, Y.-T. Liu, H. Lin, and A. Bansil, The nontrivial electronic structure of $\mathrm{Bi} / \mathrm{Sb}$ honeycombs on SiC(0001), New J. Phys. 17, 025005 (2015).

[54] E. O. Wrasse and T. M. Schmidt, Prediction of two-dimensional topological crystalline insulator in PbSe monolayer, Nano Lett. 14, 5717 (2014).

[55] J. Liu, X. Qian, and L. Fu, Crystal field effect induced topological crystalline insulators in monolayer IV-VI semiconductors, Nano Lett. 15, 2657 (2015).

[56] W. Wan, Y. Yao, L. Sun, C.-C. Liu, and F. Zhang, Topological, valleytronic, and optical properties of monolayer $\mathrm{PbS}$, Adv. Mater. 29, 1604788 (2017).

[57] B. A. Bernevig, T. L. Hughes, and S.-C. Zhang, Quantum spin Hall effect and topological phase transition in $\mathrm{HgTe}$ quantum wells, Science 314, 1757 (2006).

[58] G. R. Stewart, Superconductivity in iron compounds, Rev. Mod. Phys. 83, 1589 (2011).

[59] P. J. Hirschfeld, M. M. Korshunov, and I. I. Mazin, Gap symmetry and structure of Fe-based superconductors, Rep. Prog. Phys. 74, 124508 (2011).

[60] P. Zhang, K. Yaji, T. Hashimoto, Y. Ota, T. Kondo, K. Okazaki, Z. Wang, J. Wen, G. D. Gu, H. Ding, and S. Shin, Observation of topological superconductivity on the surface of an iron-based superconductor, Science 360, 182 (2018).

[61] D. Wang, L. Kong, P. Fan, H. Chen, S. Zhu, W. Liu, L. Cao, Y. Sun, S. Du, J. Schneeloch, R. Zhong, G. Gu, L. Fu, H. Ding, and H.-J. Gao, Evidence for Majorana bound states in an iron-based superconductor, Science 362, 333 (2018).

[62] P. Zhang, Z. Wang, X. Wu, K. Yaji, Y. Ishida, Y. Kohama, G. Dai, Y. Sun, C. Bareille, K. Kuroda et al., Multiple topological states in iron-based superconductors, Nat. Phys. 15, 41 (2019).

[63] Hard-wall boundary conditions are applicable because of the quadratic terms in our low-energy model.

[64] S.-B. Zhang, H.-Z. Lu, and S.-Q. Shen, Linear magnetoconductivity in an intrinsic topological Weyl semimetal, New J. Phys. 18, 053039 (2016)

[65] For convenience, we assume the pairing potential $\Delta(\mathbf{k})$ independent of chemical potential $\mu$. This should be justified since $\Delta(\mathbf{k})$ is induced via the proximity effect.

[66] See Supplemental Material at http://link.aps.org/supplemental/ 10.1103/PhysRevResearch.2.012018 for the derivation of the effective edge model, calculations of the supercurrent and energy spectrum of Andreev bound states in the Josephson junctions, the symmetries and the quadrupole moment of the SOTS, and further details about numerical calculations.

[67] R. Jackiw and C. Rebbi, Solitons with fermion number $1 / 2$, Phys. Rev. D 13, 3398 (1976).

[68] W. A. Benalcazar, B. A. Bernevig, and T. L. Hughes, Quantized electric multipole insulators, Science 357, 61 (2017).

[69] W. A. Benalcazar, B. A. Bernevig, and T. L. Hughes, Electric multipole moments, topological multipole moment pumping, and chiral hinge states in crystalline insulators, Phys. Rev. B 96, 245115 (2017).

[70] Z. Song, Z. Fang, and C. Fang, $(d-2)$-Dimensional Edge States of Rotation Symmetry Protected Topological States, Phys. Rev. Lett. 119, 246402 (2017).

[71] The bulk gap is given by $m_{\mathrm{g}}=m_{0}$ if $v_{x(y)}^{2}>2 m_{0} m_{x(y)}$ and $m_{\mathrm{g}}=$ $\left|v_{x(y)}\right|\left[m_{0} / m_{x(y)}-v_{x(y)}^{2} / 4 m_{x(y)}^{2}\right]^{1 / 2}$ otherwise.

[72] $\Delta_{\text {eff }}^{x(y)}$ can also switch sign at a $\mu$ larger than the bulk gap, which happens in the case of QSHI with small inverted gaps $2 m_{0} m<v^{2}$. In this case, the edge states coexist with bulk states, and Majorana corner states persist even if the bulk is not insulating.

[73] C. Liu, T. L. Hughes, X.-L. Qi, K. Wang, and S.-C. Zhang, Quantum Spin Hall Effect in Inverted Type-II Semiconductors, Phys. Rev. Lett. 100, 236601 (2008).

[74] I. Knez, R. R. Du, and G. Sullivan, Evidence for Helical Edge Modes in Inverted InAs/GaSb Quantum Wells, Phys. Rev. Lett. 107, 136603 (2011).

[75] S. S. Krishtopenko and F. Teppe, Quantum spin Hall insulator with a large bandgap, Dirac fermions, and bilayer graphene analog, Sci. Adv. 4, eaap7529 (2018).

[76] In the customary regularization, we replace $k_{x(y)} \rightarrow \sin k_{x(y)}$ and $k_{x(y)}^{2} \rightarrow 2\left[1-\cos k_{x(y)}\right]$ in the model (1) and obtain a tightbinding lattice model. We also perform the calculation for a different lattice model and obtain the same features (see the Supplemental Material)

[77] Y. Asano, Numerical method for dc Josephson current between $d$-wave superconductors, Phys. Rev. B 63, 052512 (2001).

[78] A. Martín-Rodero, F. J. García-Vidal, and A. Levy Yeyati, Microscopic Theory of Josephson Mesoscopic Constrictions, Phys. Rev. Lett. 72, 554 (1994). 
[79] A. Furusaki, DC Josephson effect in dirty SNS junctions: Numerical study, Physica B 203, 214 (1994).

[80] L. Fu and C. L. Kane, Josephson current and noise at a superconductor/quantum-spin-Hall-insulator/superconductor junction, Phys. Rev. B 79, 161408(R) (2009).

[81] F. Crépin and B. Trauzettel, Parity Measurement in Topological Josephson Junctions, Phys. Rev. Lett. 112, 077002 (2014).

[82] C.-A. Li, S.-B. Zhang, and S.-Q. Shen, Hidden edge Dirac point and robust quantum edge transport in InAs/GaSb quantum wells, Phys. Rev. B 97, 045420 (2018).

[83] C. W. J. Beenakker, Universal Limit of Critical-Current Fluctuations in Mesoscopic Josephson Junctions, Phys. Rev. Lett. 67, 3836 (1991).

[84] L. Fu and C. L. Kane, Superconducting Proximity Effect and Majorana Fermions at the Surface of a Topological Insulator, Phys. Rev. Lett. 100, 096407 (2008).
[85] R. M. Lutchyn, J. D. Sau, and S. Das Sarma, Majorana Fermions and a Topological Phase Transition in Semiconductor-Superconductor Heterostructures, Phys. Rev. Lett. 105, 077001 (2010).

[86] J. D. Sau, R. M. Lutchyn, S. Tewari, and S. Das Sarma, Generic New Platform for Topological Quantum Computation Using Semiconductor Heterostructures, Phys. Rev. Lett. 104, 040502 (2010).

[87] Y. Oreg, G. Refael, and F. von Oppen, Helical Liquids and Majorana Bound States in Quantum Wires, Phys. Rev. Lett. 105, 177002 (2010).

[88] C. Liu and S.-C. Zhang, Models and materials for topological insulators, in Contemporary Concepts of Condensed Matter Science (Elsevier, Amsterdam, 2013), Vol. 6, pp. 59-89.

[89] This corresponds to a specific axis of the crystal as discussed in Ref. [45]. 